\title{
Representations and Inequalities for Ising Model Ursell Functions
}

\author{
Garrett S. Sylvester* \\ Department of Mathematics, MIT, Cambridge, Mass., Department of Physics, \\ Harvard University, Cambridge, Mass., USA
}

Received January 13, 1975

\begin{abstract}
We describe and investigate representations for the Ursell function $u_{n}$ of a family of $n$ random variables $\left\{\sigma_{i}\right\}$. The representations involve independent but identically distributed copies of the family. We apply one of these representations in the case that the random variables are spins of a finite ferromagnetic Ising model with quadratic Hamiltonian to show that $(-1)^{\frac{n}{2}+1} u_{n}\left(\sigma_{1}, \ldots, \sigma_{n}\right) \geqq 0$ for $n=2,4$, and 6 by proving the stronger statement $\left.(-1)^{\frac{n}{2}+1} \frac{\partial^{m}}{\partial J_{i_{1} j_{1}} \cdots \partial J_{i_{m} j_{m}}} Z^{\frac{n}{2}} u_{n}\right|_{J=0} \geqq 0$ for $n=2,4$, and 6 , the $J_{i,}$ being coupling constants in the Hamiltonian and $Z$ the partition function. For general $n$ we combine this result with various reductions to show that sufficiently simple derivatives of $(-1)^{\frac{n}{2}+1} Z^{\frac{n}{2}} u_{n}$, evaluated at zero coupling, are nonnegative. In particular, we conclude that $(-1)^{\frac{n}{2}+1} u_{n} \geqq 0$ if all couplings are nonzero and the inverse temperature $\beta$ is sufficiently small or sufficiently large, though this result is not uniform in the order $n$ or the system size. In an appendix we give a simple proof of recent inequalities which bound $n$-spin expectations by sums of products of simpler expectations.
\end{abstract}

\section{Introduction}

The Ursell function $u_{n}\left(\sigma_{1}, \ldots, \sigma_{n}\right)$ of a family $\left\{\sigma_{i}\right\}$ of $n$ arbitrary random variables may be defined by means of a generating function as

$$
u_{n}\left(\sigma_{1}, \ldots, \sigma_{n}\right)=\left.\frac{\partial^{n}}{\partial \lambda_{1} \cdots \partial \lambda_{n}} \ln \mathscr{E}\left(\exp \left[\sum_{i=1}^{n} \lambda_{i} \sigma_{i}\right]\right)\right|_{\lambda=0}
$$

Here $\mathscr{E}$ is the expectation integral; we assume all the necessary expectations are finite. The Ursell function may be defined recursively by

$$
\mathscr{E}\left(\sigma_{1} \sigma_{2} \cdots \sigma_{n}\right)=\sum_{\mathscr{P}} \prod_{P \in \mathscr{P}} u_{|P|}\left(\sigma_{p_{a}}, \sigma_{p_{b}}, \ldots\right)
$$

Here $\mathscr{P}$ is a partition of $\{1, \ldots, n\}$, a set $P \in \mathscr{P}$ has elements $p_{a}, p_{b}$, etc., and $|P|$ denotes the cardinality of $P$. Finally, $u_{n}\left(\sigma_{1}, \ldots, \sigma_{n}\right)$ may be defined explicitly by

$$
u_{n}\left(\sigma_{1}, \ldots, \sigma_{n}\right)=\sum_{\mathscr{P}}(-1)^{|\mathscr{P}|-1}(|\mathscr{P}|-1) ! \prod_{P \in \mathscr{P}} \mathscr{E}\left(\prod_{p \in P} \sigma_{p}\right)
$$

where again $\mathscr{P}$ is a partition of $\{1, \ldots, n\}$.

* MIT Allen Fellow in Mathematics.

Supported in part by the National Science Foundation under Grant MPS 73-05037. 
In Section 2 of this paper we describe and investigate certain representations for the Ursell function of a family $\left\{\sigma_{i}\right\}$ which involve independent but identically distributed copies of the family. The use of such copies recently has been emphasized by Ginibre [1], Lebowitz [4], and Percus [6]. Let $\left\{\sigma_{i}^{\alpha}\right\}, \alpha \in\{0,1, \ldots, n-1\}$, be a collection of $n$ independent but identically distributed copies of the family $\left\{\sigma_{i}\right\}$, let $\omega$ be a primitive $n^{\text {th }}$ root of unity, and define

We shall find that

$$
s_{i}=\sum_{\alpha=0}^{n-1} \omega^{\alpha} \sigma_{i}^{\alpha} .
$$

$$
u_{n}\left(\sigma_{1}, \ldots, \sigma_{n}\right)=\frac{1}{n} \mathscr{E}\left(s_{1} s_{2} \cdots s_{n}\right),
$$

a result previously obtained in another way by Cartier (unpublished).

In the event that the family $\left\{\sigma_{i}\right\}$ has even symmetry (i.e. the expectation of the product of an odd number of $\sigma$ 's is 0 ) and $n$ is even we can cut the number of copies in half. Defining

$$
t_{i}=\sum_{\alpha=0}^{n / 2-1} \omega^{\alpha} \sigma_{i}^{\alpha}
$$

we find the simplified representation

$$
u_{n}\left(\sigma_{1}, \ldots, \sigma_{n}\right)=\frac{2}{n} \mathscr{E}\left(t_{1} t_{2} \cdots t_{n}\right) .
$$

[Of course, if the family $\left\{\sigma_{i}\right\}$ has even symmetry and $n$ is odd, $u_{n}\left(\sigma_{1}, \ldots, \sigma_{n}\right)=0$.] Finally, we indicate a manner in which additional representations may be produced at will.

The random variables in which we are primarily interested are the spin variables of a finite ferromagnetic Ising model with two-body forces. We describe such a model. The set underlying the probability space of an $N$-site model is $\Omega=\chi_{i=1}^{N}\{-1,+1\}$. The spin variable $\sigma_{i}$ is the projection of $\Omega$ onto its $i^{\text {th }}$ factor, the index $i$ commonly being called a site. The measure on $\Omega$ assigns to each point $\omega \in \Omega$ the weight $Z^{-1} \exp \left[\beta \sum_{i, j=1, i \neq j}^{N} J_{i j} \sigma_{i}(\omega) \sigma_{j}(\omega)\right]$. Here the partition function $Z$ is just the normalization factor $Z=\sum_{\omega \in \Omega} \exp \left[\beta \sum_{i \neq j} J_{i j} \sigma_{i}(\omega) \sigma_{j}(\omega)\right], \beta$ is a nonnegative parameter representing inverse temperature, and the coupling constants $J_{i j}$ (also called bonds) are nonnegative real numbers. Notice that the spin variables $\sigma_{i}$ have the even symmetry mentioned in the previous paragraph.

It has been conjectured that the Ursell functions $u_{n}\left(\sigma_{k_{1}}, \ldots, \sigma_{k_{n}}\right)$ of the spin variables of a finite ferromagnetic Ising model with two-body forces obey the inequality

$$
(-1)^{\frac{n}{2}+1} u_{n}\left(\sigma_{k_{1}}, \ldots, \sigma_{k_{n}}\right) \geqq 0 .
$$

This has been known for a long time in the case $n=2$; it is a special case of the second Griffiths inequality [2]. For $n=4,(1.8)$ follows from the GHS inequality [3]. A family of inequalities related to the $n=4$ case has been proved by Lebowitz [4]. Cartier has announced a proof of the case $n=6$. In Section 3 we use the 
representation (1.7) to give a unified proof that (1.8) holds for $n=2,4$, and 6 . A recent independent proof was given by Percus [6].

In doing so we actually establish the stronger statement

$$
(-1)^{\frac{n}{2}+1} \frac{\partial^{m}}{\partial J_{i_{1} j_{1}} \ldots \partial J_{i_{m} j_{m}}} Z^{\frac{n}{2}} u_{n}\left(\sigma_{k_{1}}, \ldots, \sigma_{k_{n}}\right) \geqq 0 .
$$

It is our conjecture that (1.9) holds for all $n$. However, it does not seem that the proof given for the low-order cases generalizes immediately. In Section 4 we present partial results showing that any derivative

$$
\left.(-1)^{\frac{n}{2}+1} \frac{\partial^{m}}{\partial J_{i_{1} j_{1}} \ldots \partial J_{i_{m} j_{m}}} Z^{\frac{n}{2}} u_{n}\right|_{J=0}
$$

which is sufficiently simple in a certain sense is nonnegative. (Of course, by evaluating at $J=0$ we mean evaluating at $J_{i j}=0 \forall i, j$.) In particular, we conclude that if every $J_{i j}$ is strictly positive, (1.8) holds for $\beta$ sufficiently small or sufficiently large.

In an appendix we give a simple proof of a family of inequalities recently obtained by Newman [5] related to those of Lebowitz [4]. These inequalities, which include the $u_{4}$ inequality, bound $n$-spin expectations by sums of products of simpler expectations.

\section{Representing an Ursell Function as an Expectation}

We describe and analyze representations for the Ursell function of a family of random variables $\left\{\sigma_{i}\right\}_{i \in\{1, \ldots, n\}}$. These representations employ independent but identically distributed copies of the original family. Let $\left\{\sigma_{i}^{\alpha}\right\}, \alpha \in\{0,1, \ldots, c\}$, be $(c+1)$ such independent copies of the family $\left\{\sigma_{i}\right\}$, each copy having the same joint distributions as $\left\{\sigma_{i}\right\}$. Given a set of coefficients $S_{i \alpha} \in \mathbb{C}$ we may define a new family of random variables $\left\{s_{i}\right\}_{i \in\{1, \ldots, n\}}$ by $s_{i}=\sum_{\alpha=0}^{c} S_{i \alpha} \sigma_{i}^{\alpha}$. We shall see that up to a simple factor the family $\left\{s_{i}\right\}$ has the same Ursell function as the original family $\left\{\sigma_{i}\right\}$. By judicious choice of the transformation coefficients $S_{i \alpha}$ we may cause all but the leading term in the Ursell function of the family $\left\{s_{i}\right\}$ to vanish, thereby transforming an Ursell function into an expectation. In the event that the family $\left\{\sigma_{i}\right\}$ has an even symmetry the representation simplifies, the number of copies employed being halved.

To exhibit the proportionality between the Ursell functions of $\left\{\sigma_{i}\right\}$ and $\left\{s_{i}\right\}$ we recall that if a family of random variables may be split into two mutually independent subfamilies, its Ursell function vanishes. [This is immediate from definition (1.1) because the expectation factors.] Thus,

$$
\begin{aligned}
u_{n}\left(S_{1}, \ldots, S_{n}\right) & =\sum_{\alpha_{1}, \ldots, \alpha_{n}} S_{1 \alpha_{1}} \ldots S_{n \alpha_{n}} u_{n}\left(\sigma_{1}^{\alpha_{1}}, \ldots, \sigma_{n}^{\alpha_{n}}\right) \\
& =\left\{\sum_{\alpha=0}^{c} S_{1 \alpha} \ldots S_{n \alpha}\right\} \cdot u_{n}\left(\sigma_{1}, \ldots, \sigma_{n}\right)
\end{aligned}
$$

since only those terms for which $\alpha_{1}=\alpha_{2}=\cdots=\alpha_{n}$ survive. 
Next we give a specific choice for the transformation coefficients $S_{i \alpha}$ such that $u_{n}\left(s_{1}, \ldots, s_{n}\right)=\mathscr{E}\left(s_{1} s_{2} \ldots s_{n}\right)$. Take $n$ copies of the original family $\left\{\sigma_{i}\right\}$, and for $S_{i \alpha}$ choose $\omega^{\alpha}, \omega$ being a primitive $n^{\text {th }}$ root of unity. Thus we have

$$
s_{i}=\sum_{\alpha=0}^{n-1} \omega^{\alpha} \sigma_{i}^{\alpha} .
$$

We claim that $\mathscr{E}\left(s_{1} \ldots s_{k}\right)=0$ unless $k \equiv 0(n)$. In establishing this it is convenient to regard the superscripts $\alpha$ as running through the elements of $\mathbb{Z}_{n}$. Notice that $\mathscr{E}\left(\sigma_{1}^{\alpha_{1}} \ldots \sigma_{k}^{\alpha_{k}}\right)$ is unaltered if we subtract (in $\left.\mathbb{Z}_{n}\right)$ the same constant $\beta \in \mathbb{Z}_{n}$ from each $\alpha_{i}$. Thus,

$$
\begin{aligned}
\mathscr{E}\left(s_{1} \ldots s_{k}\right) & =\sum_{\alpha_{1}, \cdots, \alpha_{k} \in \mathbb{Z}_{n}} \omega^{\alpha_{1}+\cdots+\alpha_{k}} \mathscr{E}\left(\sigma_{1}^{\alpha_{1}} \ldots \sigma_{k}^{\alpha_{k}}\right) \\
& =\sum_{\alpha_{1}, \ldots, \alpha_{k} \in \mathbb{Z}_{n}} \omega^{\alpha_{1}+\cdots+\alpha_{k}} \mathscr{E}\left(\sigma_{1}^{\alpha_{1}-\beta} \ldots \sigma_{k}^{\alpha_{k}-\beta}\right) \\
& =\sum_{\alpha_{1}, \ldots, \alpha_{k} \in \mathbb{Z}_{n}} \omega^{\alpha_{1}+\cdots+\alpha_{k}+k \beta} \mathscr{E}\left(\sigma_{1}^{\alpha_{1}} \ldots \sigma_{k}^{\alpha_{k}}\right) \\
& =\frac{1}{n} \sum_{\alpha_{1}, \ldots, \alpha_{k}, \beta \in \mathbb{Z}_{n}} \omega^{\alpha_{1}+\cdots+\alpha_{k}+k \beta} \mathscr{E}\left(\sigma_{1}^{\alpha_{1}} \ldots \sigma_{k}^{\alpha_{k}}\right) \\
& =0 \text { unless } k \equiv 0(n) \text { since } \sum_{\beta=0}^{n-1} \omega^{k \beta}=0 \text { unless } k \equiv 0(n) .
\end{aligned}
$$

I am indebted to Joel Feldman for this simple argument. With this choice of variables, we have

$$
u_{n}\left(\sigma_{1}, \ldots, \sigma_{n}\right)=\frac{1}{n} \mathscr{E}\left(s_{1} s_{2} \ldots s_{n}\right) .
$$

It may happen that the family $\left\{\sigma_{i}\right\}$ has even symmetry; that is, the expectation of any product of an odd number of $\sigma$ 's is 0 . In this case a simpler representation involving only $\frac{n}{2}$ copies of the family $\left\{\sigma_{i}\right\}$ is possible. [We take $n$ even since for odd $n$ by symmetry $u_{n}\left(\sigma_{1}, \ldots, \sigma_{n}\right)=0$.] Let

$$
t_{i}=\sum_{\alpha=0}^{\frac{n}{2}-1} \omega^{\alpha} \sigma_{i}^{\alpha},
$$

where again $\omega$ is a primitive $n^{\text {th }}$ root of unity. To apply the preceding argument to show $\mathscr{E}\left(t_{1} \ldots t_{n}\right)$ vanishes unless $k \equiv 0(n)$ we note that the superscripts $\alpha$ essentially may be regarded as elements of $\mathbb{Z}_{n / 2}$ because the ambiguity in the definition of $\omega^{\alpha_{1}+\cdots+\alpha_{k}}$ is obviated by the even symmetry of the family $\left\{\sigma_{i}\right\}$. Thus with even symmetry we find

$$
u_{n}\left(\sigma_{1}, \ldots, \sigma_{n}\right)=\frac{2}{n} \mathscr{E}\left(t_{1} t_{2} \ldots t_{n}\right) .
$$

Finally, we remark that if one chooses $S_{i \alpha}=\omega^{f_{2} \alpha}, f_{i} \in \mathbb{Z}_{n}$, only those terms $\prod_{P \in \mathscr{P}} \mathscr{E}\left(\prod_{i \in P} s_{i}\right)$ in the definition (1.3) of $u_{n}\left(s_{1}, \ldots, s_{n}\right)$ survive which satisfy the condition $\forall P \in \mathscr{P}, \sum_{i \in P} f_{i} \equiv 0(n)$. By varying the $f_{i}$, different representations for $u_{n}\left(\sigma_{1}, \ldots, \sigma_{n}\right)$ may be obtained. For example, the representations above have $f_{i}=1 \forall i$, and only the leading term survives. On the other hand, the representation used by Lebowitz [4] exploits the even symmetry as in (2.5) with $f_{1}=f_{2}=0, f_{3}=f_{4}=2$, and two terms survive. 


\section{Signs of the Ursell Functions $\boldsymbol{u}_{2}, \boldsymbol{u}_{\mathbf{4}}, \boldsymbol{u}_{\mathbf{6}}$ for Ferromagnetic Ising Models}

We employ the representation $(2.5)$ in the case where the random variables $\left\{\sigma_{i}\right\}$ are spin variables of a finite ferromagnetic Ising model with two-body forces. (See Section 1 for a definition and notation.) Using it, we show that $(-1)^{\frac{n}{2}+1} u_{n}\left(\sigma_{k_{1}}, \ldots, \sigma_{k_{n}}\right) \geqq 0$ for $n=2,4$, and 6 by proving the stronger statement that, in the ferromagnetic region, all derivatives with respect to coupling constants $J_{i j}$ of $(-1)^{\frac{n}{2}+1} Z^{\frac{n}{2}} u_{n}$ are nonnegative. The proof does not generalize immediately to the case of higher order Ursell functions, and in fact appears somewhat fortuitous. Nevertheless, we believe that in general the derivatives with respect to coupling constant of $(-1)^{\frac{n}{2}+1} Z^{\frac{n}{2}} u_{n}$ are nonnegative and this belief is supported by asymptotic results for both low and high temperature.

To use the representation (2.5) we enlarge the set underlying the probability

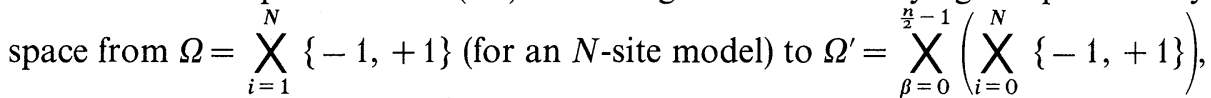
with the random variables $\sigma_{i}^{\beta}$ being the projections onto the $(\beta, i)^{\text {th }}$ factors. The (unnormalized) weight of each state is given by $\exp \left(\sum_{\beta=0}^{\frac{n}{2}-1} \sum_{i \neq j} J_{i j} \sigma_{i}^{\beta} \sigma_{j}^{\beta}\right)$. Extend the definition (2.4) of the variables $t_{i}$ by setting

$$
t_{i}^{\alpha}=\sum_{\beta=0}^{\frac{n}{2}-1} \omega^{\alpha \beta} \sigma_{i}^{\beta}, \quad \alpha \in\{1,3,5, \ldots, n-1\} .
$$

Thus what we called $t_{i}$ in (2.4) is $t_{i}^{1}$ here. Note that $\left(t_{i}^{\alpha}\right)^{*}=t_{i}^{n-\alpha}$.

For $\alpha \in\{1,3,5, \ldots, n-1\}$ and $\beta \in\left\{0,1, \ldots, \frac{n}{2}-1\right\}$ the matrix $\sqrt{\frac{2}{n}} \omega^{\alpha \beta}$ is unitary. Thus,

$$
\sum_{i, j, \beta} J_{i j} \sigma_{i}^{\beta} \sigma_{j}^{\beta}=\frac{2}{n} \sum_{i, j, \alpha} J_{i j} t_{i}^{\alpha} t_{j}^{n-a},
$$

and in the $t$-variables the representation (2.5) may be written

$$
Z^{\frac{n}{2}} u_{n}\left(\sigma_{k_{1}}, \ldots, \sigma_{k_{n}}\right)=\frac{2}{n} \operatorname{Tr}\left(t_{k_{1}}^{1} \ldots t_{k_{n}}^{1} \exp \left[\frac{2}{n} \sum_{i, j, \alpha} J_{i j} t_{i}^{\alpha} t_{j}^{n-\alpha}\right]\right) .
$$

(Given a finite set $\Omega^{\prime}$ and a function $f: \Omega^{\prime} \rightarrow \mathbb{C}$, by $\operatorname{Tr} f$ we mean $\sum_{\omega^{\prime} \in \Omega^{\prime}} f\left(\omega^{\prime}\right)$.) The derivative of (3.3) with respect to coupling constants $J_{i_{1} j_{1}}, \ldots, J_{i_{m} j_{m}}$ is

$$
\begin{aligned}
& \frac{\partial^{m}}{\partial J_{i_{1} j_{1}} \ldots \partial J_{i_{m} j_{m}}} Z^{\frac{n}{2}} u_{n}\left(\sigma_{k_{1}}, \ldots, \sigma_{k_{n}}\right) \\
& \quad=\sum_{\alpha_{1}, \ldots, \alpha_{m}}\left(\frac{2}{n}\right)^{m+1} \operatorname{Tr}\left(t_{k_{n}}^{1} \ldots t_{k_{n}}^{1} t_{i_{1}}^{\alpha_{1}} n_{j_{1}}^{n-\alpha_{1}} \ldots t_{i_{m}}^{\alpha_{m}} t_{j_{m}}^{n-\alpha_{m}} \exp \left[\sum_{i, j, \alpha} \frac{2}{n} J_{i j} t_{i}^{\alpha} t_{j}^{n-\alpha}\right]\right) .
\end{aligned}
$$

In order to show that all these derivatives have a certain sign when evaluated at arbitrary $J_{i j} \geqq 0$ is suffices to show they all have this sign when the couplings $J_{i j}$ are set to zero, and this is what we do for $n=2,4$, and 6 .

Theorem 3.1. Let $u_{n}$ be the Ursell function of a finite ferromagnetic Ising model with Hamiltonian $-\sum_{i \neq j} J_{i j} \sigma_{i} \sigma_{j}$ and partition function $Z$. For $n=2,4$, and 6 
all derivatives with respect to coupling constant of $(-1)^{\frac{n}{2}} Z^{\frac{n}{2}} u_{n}\left(\sigma_{k_{1}}, \ldots, \sigma_{k_{n}}\right)$ are nonnegative. Moreover, if every site in the model may be connected with every other site by a chain of nonzero couplings the inequality is strict.

Remark. These inequalities, which as they stand explicitly involve factors of $Z$, may be converted to inequalities involving the spins alone by dividing by $Z^{\frac{n}{2}}$.

Proof. We give the proof only for the case $n=6$. The case $n=4$ may be done in a similar way, and the case $n=2$ is trivial.

We want to show that the sum $\sum_{\alpha_{1}, \ldots, \alpha_{m}} \operatorname{Tr}\left(t_{k_{1}}^{1} \ldots t_{k_{6}}^{1} t_{i_{1}}^{\alpha_{1}} t_{j_{1}}^{n-\alpha_{1}} \ldots t_{i_{m}}^{\alpha_{m}} t_{j_{m}}^{n-\alpha_{m}}\right)$ arising from the evaluation of (3.4) at $J=0$ is nonnegative. It is actually true that an individual term $\operatorname{Tr}\left(t_{k_{1}}^{1} \ldots t_{j_{m}}^{n-\alpha_{m}}\right)$ is nonnegative. Since this trace factors over sites, we break it up into a product of traces of the form $\operatorname{Tr}\left(t^{\gamma_{1}} \ldots t^{\gamma_{a}}\right)$, with the common site subscript suppressed. By an argument given in Section 2 in connection with representations (2.2) and (2.5), this trace vanishes unless $\gamma_{1}+\cdots+\gamma_{a} \equiv 0(n)$. Assume this condition is satisfied at all sites. We claim that the function $t^{\gamma_{1}} \ldots t^{\gamma_{a}}$ obeys the inequality

$$
(-1)^{\left|\left\{i \mid y_{i}=3\right\}\right|} t^{\gamma_{1}} \ldots t^{\gamma_{a}} \geqq 0 .
$$

To see this is true, we note that since $\left(t^{1}\right)^{*}=t^{5}$ and $\left(t^{3}\right)^{*}=t^{3}$, pairing $t^{1}$ s with $t^{5}$ s and $t^{3}$ 's with one another reduces the problem to showing that $\left(t^{1}\right)^{6} \geqq 0$ and $\left(t^{1}\right)^{3} t^{3} \leqq 0$. This may be done by explicit verification of cases. It now follows immediately that the product over the sites of the terms $t^{\gamma_{1}} \ldots t^{\gamma_{a}}$ is nonnegative and so has nonnegative trace, because the total number of $\gamma$ 's appearing with value 3 is even.

The strict positivity may be seen in several ways. One simple one is to resurrect the inverse temperature $\beta$. Note that if a finite ferromagnetic Ising model is connected by nonzero couplings as in the hypothesis of the theorem, then for any function of the spins $F\left(\sigma_{1}, \ldots, \sigma_{N}\right)$

$$
\lim _{\beta \rightarrow \infty} \mathscr{E}(F)=\frac{1}{2}[F(-1, \ldots,-1)+F(1, \ldots, 1)] .
$$

Thus in such a model, $\lim _{\beta \rightarrow \infty} Z^{-3} \frac{\partial^{m}}{\partial J_{i_{1} j_{1}} \ldots \partial J_{i_{m j} j_{m}}} Z^{3} u_{6}=3^{m} \cdot 16$. But since all the coefficients in the Maclaurin expansion of $Z^{3} u_{6}$ in the couplings are nonnegative, if the above derivative were 0 for $\beta=1$ it would remain so $\forall \beta$ and, when normalized by $Z^{3}$, could not converge to $3^{m} \cdot 16$ as $\beta \rightarrow \infty$.

Q.E.D.

We remark that by using the "ghost spin" method of Griffiths [2,3], in which the values of a (nonuniform) external field are regarded as bonds coupling the model to an additional "ghost" spin, we may extend Theorem 3.1 to the case of positive external field, provided the Ursell functions are modified by dropping all terms involving the expectation of an odd number of spins.

Next we state a corollary of this theorem, and finally comment on some aspects of this proof. The corollary extends the theorem to Ursell functions of arbitrary order, provided that at most seven distinct spin sites appear among the arguments, by means of a reduction formula. The reduction formula provides the necessary combinatorics for expressing Ursell functions with repeated spin arguments in terms of simpler Ursell functions. To state it we need some notation. 
Let $\left\{\sigma_{i}\right\}_{i \in\{1, \ldots, n\}}$ be a family of $n$ random variables, and let $\mathscr{P}, \mathscr{Q}$ be partitions of $\{1, \ldots, n\}$. Define

$$
u_{\mathscr{Q}}\left(\sigma_{1}, \ldots, \sigma_{n}\right)=\prod_{Q \in \mathscr{Q}} u_{|Q|}\left(\sigma_{q_{a}}, \sigma_{q_{b}}, \ldots\right),
$$

where $q_{a}, q_{b}$, etc. are the elements of $Q$. Define the family $\left\{\sigma_{P}\right\}_{P \in \mathscr{P}}$ of random variables by

$$
\sigma_{P}=\prod_{i \in P} \sigma_{i}
$$

Let $\mathscr{P} \vee \mathscr{Q}$ denote the finest partition coarser than both $\mathscr{P}$ and $\mathscr{Q}$, and let $\mathbb{1}$ be the one-element partition $\{\{1, \ldots, n\}\}$. A simple combinatoric calculation with Möbius functions gives the following lemma.

Lemma 3.2. Let $\left\{\sigma_{i}\right\}$ be a family of $n$ random variables. Then, with the above notation, $u_{|\mathscr{P}|}\left(\left\{\sigma_{P}\right\}\right)=\sum_{\mathscr{Q} \mid \mathscr{Q} \vee \mathscr{P}=\mathbb{1}} u_{\mathscr{Q}}\left(\sigma_{1}, \ldots, \sigma_{n}\right)$.

As a special case of this we have

$$
\begin{aligned}
& u_{n-1}\left(\sigma_{1} \cdot \sigma_{2}, \sigma_{3}, \ldots, \sigma_{n}\right) \\
& \quad=u_{n}\left(\sigma_{1}, \sigma_{2}, \ldots, \sigma_{n}\right)+\sum_{\substack{1 \in P \\
P \subset\{1, \ldots, n\} \mid 2 \notin P}} u_{k}\left(\sigma_{p_{1}}, \ldots, \sigma_{p_{k}}\right) \cdot u_{l}\left(\sigma_{q_{1}}, \ldots, \sigma_{q_{l}}\right),
\end{aligned}
$$

where $P=\left\{p_{1}, \ldots, p_{k}\right\}$ and $\hat{P}=\left\{q_{1}, \ldots, q_{l}\right\}$. If $\sigma_{1} \cdot \sigma_{2}$ is independent of the remaining random variables, as in the case when $\sigma_{1}$ and $\sigma_{2}$ are spins from the same site, the lefthand side of (3.9) is 0 [as in the derivation of (2.1)] and we obtain the reduction

$$
u_{n}\left(\sigma_{1}, \ldots, \sigma_{n}\right)=-\sum_{\substack{1 \in P \\ P \subset\{1, \ldots, n\} \mid 2 \notin P}} u_{k}\left(\sigma_{p_{1}}, \ldots, \sigma_{p_{k}}\right) u_{l}\left(\sigma_{q_{1}}, \ldots, \sigma_{q_{l}}\right) .
$$

We use this to prove

Corollary 3.3. Let $u_{n}\left(\sigma_{k_{1}}, \ldots, \sigma_{k_{n}}\right)$ be an Ursell function of the Ising model of Theorem 3.1. If the $n$ spins used as arguments are selected from at most seven different sites, then all derivatives with respect to coupling constants $J_{i j}$ of $(-1)^{\frac{n}{2}+1} Z^{\frac{n}{2}} u_{n}\left(\sigma_{k_{1}}, \ldots, \sigma_{k_{n}}\right)$ are nonnegative. Moreover, if every site in the model may be connected with every other site by a chain of nonzero couplings, the inequality is strict.

Proof. We prove this by induction on $n$. By the theorem, it is obviously true if $n \leqq 6$. If $n>6$, two spins must be selected from the same site, say $k_{1}=k_{2}$. By reduction (3.10)

and so

$$
u_{n}\left(\sigma_{k_{1}}, \ldots, \sigma_{k_{n}}\right)=-\sum_{P} u_{k}\left(\sigma_{p_{1}}, \ldots, \sigma_{p_{k}}\right) u_{l}\left(\sigma_{q_{1}}, \ldots, \sigma_{q_{l}}\right),
$$

$$
(-1)^{\frac{n}{2}+1} Z^{\frac{n}{2}} u_{n}=+\sum_{P}\left[(-1)^{\frac{k}{2}+1} Z^{\frac{k}{2}} u_{k}\right]\left[(-1)^{\frac{l}{2}+1} Z^{\frac{l}{2}} u_{l}\right]
$$

with notation as above. From (3.11) the corollary is immediate.

Again, note that the Griffiths "ghost spin" method allows immediate extension of this result to the case of positive external field if the Ursell functions are properly modified.

We now return our attention to the proof of Theorem 3.1 It proceeds by showing that the derivatives with respect to $J_{i j}$ of $(-1)^{\frac{n}{2}+1} Z^{\frac{n}{2}} u_{n}$, evaluated at zero 
coupling, are nonnegative for $n \leqq 6$. One can say explicitly which derivatives are zero and which are not. A derivative with respect to certain couplings is nonzero $\Leftrightarrow$

(1) any two sites appearing as arguments of the Ursell function may be connected by a chain of these couplings; and,

(2) for any site, the number of these couplings touching it plus the number of times it appears as an argument is even. These conditions may be interpreted conveniently in terms of the graphical notation discussed in the following section.

Finally, we comment on the restriction of the proof to the case $n \leqq 6$. In this case we established that the sum $\sum_{\alpha_{1}, \ldots, \alpha_{m}} \operatorname{Tr}\left(t_{1}^{1} \ldots t_{n}^{1} t_{i_{1}}^{\alpha_{1}} t_{j_{1}}^{n-\alpha_{1}} \ldots t_{i_{m}}^{\alpha_{m}} t_{j_{m}}^{n-\alpha_{m}}\right)$ had sign $(-1)^{\frac{n}{2}+1}$ by showing that each term in the sum had this sign. For $n>6$ this is no longer possible. For example, in the case $n=8$ it may be shown that $\operatorname{Tr}\left(\left[\left(t_{1}^{1}\right)^{7}\left(t_{2}^{1}\right)\right]\left[\left(t_{1}^{3} t_{2}^{5}\right)^{3}\right]\right)>0$. However, it is known by other reasoning that the full sum

$$
\sum_{\alpha_{1}, \alpha_{2}, \alpha_{3}} \operatorname{Tr}\left(\left[\left(t_{1}^{1}\right)^{7}\left(t_{2}^{1}\right)\right]\left[t_{1}^{\alpha_{1}} t_{2}^{3-\alpha_{1}} \ldots t_{1}^{\alpha_{3}} t_{2}^{3-\alpha_{3}}\right]\right)
$$

is negative. We conjecture that the derivatives (essentially, the full sums) have $\operatorname{sign}(-1)^{\frac{n}{2}+1}$ for arbitrary $n$. This is known for general $n$ for a fairly large class of derivatives, as discussed in the following section, and investigation is continuing.

\section{Simple Derivatives and Graphical Notation}

In this section we combine Theorem 3.1 and reduction (3.10) inductively to show that sufficiently simple derivatives with respect to coupling constants $J_{i j}$ of $(-1)^{\frac{n}{2}+1} Z^{\frac{n}{2}} u_{n}\left(\sigma_{k_{1}}, \ldots, \sigma_{k_{n}}\right)$, evaluated at $J=0$, are nonnegative. Naturally, one hopes that eventually all derivatives will be shown nonnegative.

As a preliminary, we establish a graphical notation for derivatives $\left.\frac{\partial^{m}}{\partial J_{i_{1} j_{1}} \ldots \partial J_{i_{m} j_{m}}} Z^{\frac{n}{2}} u_{n}\left(\sigma_{k_{1}}, \ldots, \sigma_{k_{n}}\right)\right|_{J=0}$ of Ursell functions. Regard the sites of our Ising model as vertices of a linear graph. For each $\frac{\partial}{\partial J_{i j}}$ appearing in the derivative put an edge between sites $i$ and $j$. This specifies the derivative. To specify the arguments $\sigma_{k_{a}}$ of $u_{n}$, introduce $n$ dummy vertices - one for each $k_{a}$-and put an edge between each site $k_{a}$ and its associated dummy vertex. Finally, suppress all vertices not touched by an edge. The resulting graph $G$ is called the graph of the derivative, and the derivative the value $[G]$ of the graph. When calculating the value of a graph it is convenient to normalize the trace so that $\operatorname{Tr}(\mathbb{1})=1$, thereby eliminating factors of $2^{N}$. As an example, the graph of

is

$$
\left.\frac{\partial^{2}}{\left(\partial J_{12}\right)^{2}} Z^{2} u_{4}\left(\sigma_{1}, \sigma_{1}, \sigma_{2}, \sigma_{2}\right)\right|_{J=0}
$$

Dummy vertices

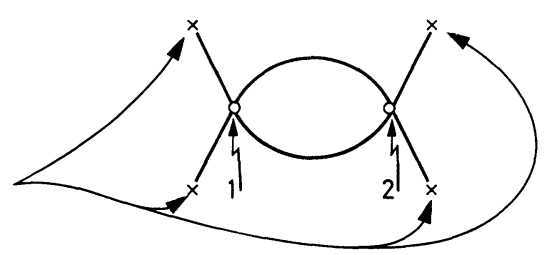

Fig. 1

and has value -4 . 
Some of the results of prior sections have pleasant interpretations in this notation. For example, evaluating (3.4) at $J=0$, we may identify each term in the sum on the right with a system of odd $\mathbb{Z}_{n}$-valued currents on the graph of the left-hand side. The current carried by an edge into a vertex is the superscript of the associated $t$-variable, and the dummy vertices are regarded as unit sources. Thus, the term $\operatorname{Tr}\left(t_{1}^{1} t_{1}^{1} t_{2}^{1} t_{2}^{1} t_{1}^{1} t_{2}^{3} t_{1}^{1} t_{2}^{3}\right)$ would be represented by the network

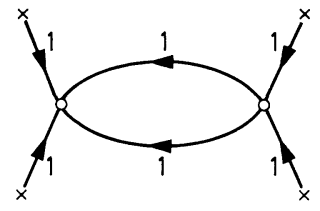

Fig. 2

As we saw in the proof of Theorem 3.1, for such a term to be nonzero the associated network must obey the Kirchoff current law in $\mathbb{Z}_{n}$. From this we conclude $\left.\frac{\partial^{m}}{\partial J_{i_{1} j_{1}} \ldots \partial J_{i_{m} j_{m}}} Z^{\frac{n}{2}} u_{n}\left(\sigma_{k_{1}}, \ldots, \sigma_{k_{n}}\right)\right|_{J=0}$ vanishes unless all the argument edges of the associated graph lie in the same connected component and the number of edges incident on each vertex (except the dummies) is even. Such graphs will be called nontrivial.

The reduction (3.10) also may be graphically interpreted. Differentiating the identity, we find that if two argument edges $e_{1}, e_{2}$ in a graph $G$ share a common vertex then

$$
[G]=-\sum_{\substack{H_{1} \cup H_{2}=G \\ H_{1} \cap H_{2}=\vartheta \\ e_{i} \in H_{i}}}\left[H_{1}\right]\left[H_{2}\right] .
$$

By this notation we mean that $H_{1}$ and $H_{2}$ are the elements of a partition $G$ into two subgraphs, with edge $e_{i}$ in subgraph $H_{i}$; the sum extends over all such partitions.

With this graphical notation we may prove

Proposition 4.1. If the graph of the derivative

$$
\left.\frac{\partial^{m}}{\partial J_{i_{1} j_{1}} \cdots \partial J_{i_{m} j_{m}}} Z^{\frac{n}{2}} u_{n}\left(\sigma_{k_{1}}, \ldots, \sigma_{k_{n}}\right)\right|_{J=0}
$$

is nontrivial and has at most four independent loops in the component of the argument edges, then $\left.(-1)^{\frac{n}{2}+1} \frac{\partial^{m}}{\partial J_{i_{1} j_{1}} \cdots \partial J_{i_{m} j_{m}}} Z^{\frac{n}{2}} u_{n}\left(\sigma_{k_{1}}, \ldots, \sigma_{k_{n}}\right)\right|_{J=0} \geqq 0$.

Proof. We use induction on the total number of edges. Since the trace factors over sites, connected components without argument edges merely contribute positive factors to the value, so we may assume that the component of the argument edges is the only component. By Theorem 3.1 we may assume at least 8 argument edges. If any two argument edges share a common vertex, we use the reduction (4.1). Also, if any argument edge is incident on a vertex with only one other incident edge, we may simply erase the argument edge and call the other edge an argument edge without changing the value of the graph. There remains only the case in which each argument edge shares a vertex with at least three other edges, all of which must be derivative edges. 
We claim that in this situation with at most four independent loops there can be at most six argument edges. We restrict our attention to the subgraph $G^{\prime}$ of $G$ which contains only the derivative edges; let it have $E^{\prime}$ edges and $V^{\prime}$ vertices. The number of independent loops $\lambda^{\prime}$ is

$$
\lambda^{\prime}=E^{\prime}-V^{\prime}+1 \text {. }
$$

Of course, this number is the same for $G$ and $G^{\prime}$. With the restrictions in the case at hand, we see easily that

consequently

$$
E^{\prime} \geqq \frac{1}{2}\left(3 n+2\left[V^{\prime}-n\right]\right)=\frac{n}{2}+V^{\prime} ;
$$

which verifies the claim.

$$
\lambda^{\prime} \geqq \frac{n}{2}+1
$$

Combining this proposition with Corollary 3.3, we may say that derivatives of $Z^{\frac{n}{2}} u_{n}$ have the conjectured sign provided either they are simple in not having argument edges at too many vertices in the associated graph, or in not having graphs which are too connected.

With a little more work, one may show that the inequality in Proposition 4.1 is actually strict. Thus we have the asymptotic result

Corollary 4.2. Let $u_{n}\left(\sigma_{k_{1}}, \ldots, \sigma_{k_{n}}\right)$ be an Ursell function of a finite ferromagnetic Ising model with two-body forces and all couplings $J_{i j}$ nonzero. Then, if the inverse temperature $\beta$ is sufficiently small or sufficiently large, $(-1)^{\frac{n}{2}+1} u_{n}\left(\sigma_{k_{1}}, \ldots, \sigma_{k_{n}}\right) \geqq 0$.

Proof. For small $\beta$, expand $Z^{\frac{n}{2}} u_{n}\left(\sigma_{k_{1}}, \ldots, \sigma_{k_{n}}\right)$ as a power series in $\beta J_{i j}$. We may use the reduction. (3.10) to assume the sites $k_{1}, \ldots, k_{n}$ are distinct. For distinct sites, the lowest-order nonzero graphs are trees, which by Proposition 4.1 have the claimed sign.

For large $\beta$ we use (3.6) to conclude $\left.u_{n}\left(\sigma_{k_{1}}, \ldots, \sigma_{k_{n}}\right) \rightarrow \frac{d^{n}}{d \lambda^{n}} \ln \cosh \lambda\right|_{\lambda=0}$ as $\beta \rightarrow \infty$. This derivative has the asserted sign.

Methods for explicit calculation of graphs are available. One method evaluates graphs with currents as in Fig. 4.2. Another sums over the derivative currents and evaluates graphs of the type in Fig. 4.1. In either case, what results is a sum of products of hyperbolic tangent numbers $\left.\frac{d^{m}}{d \lambda^{m}} \tanh \lambda\right|_{\lambda=0}$. For example, a tree with a set $\mathscr{V}^{\prime}$ of non-dummy vertices has value $\left.\prod_{v \in \mathscr{V}^{\prime}} \frac{d^{|\mathscr{V}|-1}}{d \lambda^{|\mathscr{V}|-1}} \tanh \lambda\right|_{\lambda=0}-$ only one term in the sum. Here $|v|$ is the number of edges incident on vertex $v$. Generally, the more connected the graph, the more complicated the computation.

Using these calculation methods, various graphs not reducible in the manner of Proposition 4.1 or Corollary 3.3 have been evaluated in a computer study. They all have the conjectured sign.

\section{Appendix}

We use a method originally devised for showing $u_{4}$ negative to give a simple proof of a family of inequalities recently obtained by Newman [5] which is closely related to the inequalities of Lebowitz [4]. 
First we define admissibility. Let $K$ be a finite set of even cardinality. Use ${ }^{\sim}$ to denote complementation in $K$. A collection $\mathscr{A}$ of even subsets of $K$ is admissible if and only if every partition of $K$ into pairs is a refinement of some two-element partition $\{A, \tilde{A})$ with $A \in \mathscr{A}$.

Theorem A.1. Let $K$ be an even set of sites in a finite ferromagnetic Ising model with two-body forces and nonnegative (nonuniform) external field. If the collection $\mathscr{A}$ of subsets of $K$ is admissible then

$$
\left\langle\sigma_{K}\right\rangle \leqq \sum_{A \in \mathscr{A}}\left\langle\sigma_{A}\right\rangle\left\langle\sigma_{\tilde{A}}\right\rangle
$$

(Here $\langle$,$\rangle is used to denote thermal average, and given a set of sites B$ we define $\sigma_{B}=\prod_{b \in B} \sigma_{b}$.)

Proof. By the "ghost spin" method of Griffiths [2, 3] it suffices to consider the case of zero external field. In this case we claim that all derivatives with respect to coupling constants of $Z^{2}\left(\left\langle\sigma_{K}\right\rangle-\sum_{\mathscr{A}}\left\langle\sigma_{A}\right\rangle\left\langle\sigma_{\tilde{A}}\right\rangle\right)$ are nonpositive when evaluated at zero coupling, and hence throughout the ferromagnetic region.

It is convenient to represent a differential operator $D=\frac{\partial^{m}}{\partial J_{i_{1} j_{1}} \ldots \partial J_{i_{m} j_{m}}}$ in the coupling constants by a graph $\Gamma$. The vertices of $\Gamma$ are sites in the model and for each derivative $\frac{\partial}{\partial J_{i j}}$ appearing in $D$ we place an edge between vertices (sites) $i$ and $j$. Sites with no incident edges are then suppressed. For example, the differential operator $\frac{\partial^{4}}{\partial J_{12} \partial J_{12} \partial J_{13} \partial J_{23}}$ would be represented by the graph

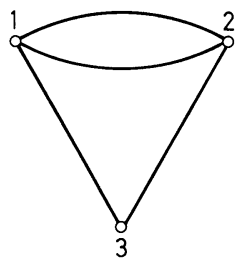

Fig. 3

To simplify the notation, given a set of sites $B$ we write $[B]$ for $\operatorname{Tr}\left(\sigma_{B} e^{-\beta H}\right)$ and $\Gamma \cdot[B]$ for the action of the derivative associated with the graph $\Gamma$ on $\operatorname{Tr}\left(\sigma_{B} e^{-\beta H}\right)$. Finally, define the ( $\mathbb{Z}_{2}$-reduced) boundary $\partial \Gamma$ of a graph $\Gamma$ to be the set of all vertices of $\Gamma$ having an odd number of incident edges.

With this notation our claim becomes

$$
\left.\Gamma \cdot\left([\phi][K]-\sum_{\mathscr{A}}[A][\tilde{A}]\right)\right|_{J=0} \leqq 0
$$

for all derivative graphs $\Gamma$, and it is a consequence of the following three statements.

(1) $\left.\Gamma \cdot([\phi][K])\right|_{J=0}$ and $\left.\Gamma \cdot([A][\hat{A}])\right|_{J=0}$ both vanish unless $\partial \Gamma=K$.

(2) If $\partial \Gamma=K$ then there exists a subgraph $G$ of $\Gamma$ and a set $A \in \mathscr{A}$ with $\partial G=A$.

(3) $G \cdot([\phi][K]-[A][\tilde{A}]) \equiv 0$, so $\Gamma \cdot([\phi][K]-[A][\tilde{A}]) \equiv 0$. 
Since the remaining terms on the left of the inequality are manifestly negative, this cancellation verifies the claim.

Statement (1) is obvious.

Statement (2) is a straightforward induction. Since $\partial \Gamma=K$, given a site $k \in K \exists$ site $k^{\prime} \in K$ connected with $k$ by some path $\gamma$ in $\Gamma$. After removing $k$ and $k^{\prime}$ from $K$ and $\gamma$ from $\Gamma$, we see that by repeating the argument we may produce a partition of $K$ into pairs $\left\{k, k^{\prime}\right\}$ connected in $\Gamma$ by edge-disjoint paths $\gamma$. Since $\mathscr{A}$ is admissible $\exists A \in \mathscr{A}$ which is a union of some of these pairs; for $G$ we just take the paths $\gamma$ connecting them.

Statement (3) is a simple calculation. Using $\Delta$ for symmetric difference we find

$$
\begin{aligned}
G \cdot([\phi][K]) & =\sum_{G_{1} \oplus G_{2}=G}\left(G_{1} \cdot[\phi]\right)\left(G_{2} \cdot[K]\right) \\
& =\Sigma\left[\partial G_{1}\right]\left[\left(\partial G_{2}\right) \Delta K\right] \\
& =\Sigma\left[\left(\partial G_{2}\right) \Delta A\right]\left[\left(\partial G_{1}\right) \Delta \tilde{A}\right] \\
& =G \cdot([A][\tilde{A}])
\end{aligned}
$$

since $\partial G_{1}=\left(\partial G_{2}\right) \Delta A$ and $\left(\partial G_{2}\right) \Delta K=\left(\partial G_{1}\right) \Delta \hat{A}$.

Corollary A.2. Let $\mathscr{C}$ be the set of all partitions of $K$ into pairs. Then

$$
\left\langle\sigma_{K}\right\rangle \leqq \sum_{\mathscr{P} \in \mathscr{C}} \prod_{\left\{k, k^{\prime}\right\} \in \mathscr{P}}\left\langle\sigma_{k} \sigma_{k^{\prime}}\right\rangle .
$$

Proof. This is immediate from successive applications of Theorem A.1. Q.E.D.

Acknowledgements. I would like to thank Professor Arthur Jaffe for suggesting this problem to me and giving encouragement and guidance towards its solution. I would like to thank Joel Feldman for many hours of useful discussion. Finally, I would like to thank Professor Pierre Cartier for telling me of his results.

\section{References}

1. Ginibre, J.: General formulation of Griffiths' inequalities. Commun. math. Phys. 16, 310-328 (1970)

2. Griffiths, R. B.: Correlations in ising ferromagnets I and II. J. Math. Phys. 8, 478-483 and 484-489 (1967)

3. Griffiths, R.B., Hurst, C.A., Sherman, S.: Concavity of magnetization of an ising ferromagnet in a positive external field. J. Math. Phys. 11, 790-795 (1970)

4. Lebowitz, J. L.: GHS and other inequalities. Commun. math. Phys. 35, 87-92 (1974)

5. Newman, C.: Gaussian Correlation Inequalitıes for Ferromagnets. Indiana Univ. preprint

6. Percus, J. K.: Correlation inequalities for ising spin lattices. Commun. math. Phys. 40, 283-308 (1975)

Communicated by G. Gallavotti
G. S. Sylvester

Department of Mathematics

MIT

Cambridge, Mass. 02139, USA 